\title{
Prevalência da retinopatia diabética em unidade do Programa de Saúde da Família
}

\author{
Prevalence of the diabetic retinopathy \\ in a Family's Health Program unity
}

Murielem Fernandes Guedes ${ }^{1}$, Arlindo José Freire Portes², Abelardo de Souza Couto Junior ${ }^{3}$, Juliana dos Santos Nunes $^{4}$, Regina Célia Coutinho Oliveira ${ }^{5}$

\begin{abstract}
Resumo
Objetivo: Determinar a prevalência da retinopatia diabética na população cadastrada no Programa de Saúde da Família (PSF) - Parque Prazeres, em Campos dos Goytacazes, RJ, e o nível de conhecimento desta doença pelos pacientes diabéticos. Métodos: Estudo descritivo de série de casos onde foram convidados a participar do trabalho de pesquisa todos os 72 pacientes diabéticos cadastrados através da ficha A (ficha de cadastro das famílias) do PSF estudado. Para a realização do estudo aceitaram participar 46 (64\%) dos 72 pacientes. Os pacientes foram submetidos ao exame de acuidade visual, sendo posteriormente aplicado um questionário para a avaliação do nível de conhecimento das alterações visuais causadas pelo diabetes, em seguida foi realizado o exame de fundoscopia direta. O estudo estatístico foi feito com auxílio do programa Microsoft Excel e Bioestat 5.0 Resultados: A prevalência da retinopatia diabética foi de 19,5\% ( IC $95 \%$ entre 8,1 e $31 \%$ ); $13 \%$ possuíam retinopatia diabética não proliferativa e $6,5 \%$ retinopatia diabética proliferativa. De acordo com critério da Organização Mundial de Saúde: $8,5 \%$ foram considerados cegos e $8,9 \%$ com baixa visão no momento do exame ocular. Setenta e dois por cento dos pacientes afirmaram ter conhecimento que o diabetes poderia causar alterações visuais, mas $80 \%$ não sabiam dos exames necessários para diagnóstico e $65 \%$ nunca foram anteriormente submetidos ao exame de fundoscopia. Conclusão: Programas de prevenção à cegueira pela retinopatia diabética no PSF são justificados, considerando que a prevalência desta doença nas comunidades carentes assistidas pode estar em níveis elevados, como a observada neste estudo. A melhora do conhecimento dos pacientes sobre o risco de perda de visão e exames oftalmológicos periódicos poderá contribuir para um melhor controle da retinopatia diabética.
\end{abstract}

Descritores: Diabetes Mellitus/complicações; Retinopatia diabética; Programa Saúde da Família;Cegueira/prevenção \& controle; Técnicas de diagnóstico oftalmológico; Oftalmoscopia/métodos

\footnotetext{
Mestre em Saúde da Família pela Universidade Estácio de Sá - Rio de Janeiro (RJ), Brasil;

2Doutor, professor Adjunto de Oftalmologia da faculdade de Medina da Universidade Estácio de Sá - Rio de Janeiro(RJ), Brasil;

${ }^{3}$ Doutor, professor Adjunto de Oftalmologia da Faculdade de Medicina de Valença (RJ), Brasil;

${ }^{4}$ Acadêmica do sexto ano da Faculdade de Medicina da Universidade Estácio de Sá - Rio de Janeiro (RJ), Brasil;

${ }^{5}$ Acadêmica do sexto ano da faculdade de Medicina da Universidade Estácio de Sá - Rio de Janeiro (RJ), Brasil.
}

Recebido para publicação em: 17/6/2008 - Aceito para publicação em 4/3/2009 


\section{INTRODUÇÃO}

$\mathbf{N}$ o Brasil, estima-se que a prevalência do diabetes melitus (DM) seja do tipo I ou II, varie de $3 \%$ a 17\% nas faixas etárias de 30-39 e de 60-69 anos ${ }^{(1)}$. Cerca de $50 \%$ dos pacientes não conhecem o diagnóstico e 24 \% que são portadores de DM não fazem nenhum tipo de tratamento. Cerca de 177 milhões de pessoas sofrem de diabetes no mundo e esse índice deverá dobrar até $2030^{(2)}$.

A estratégia do Programa Saúde da Família (PSF) incorpora e reafirma os princípios básicos do SUS universalização, descentralização, integralidade e participação da comunidade. Está estruturada a partir da Unidade Básica de Saúde da Família (UBSF) com bases nos seguintes princípios: integralidade, hierarquização, territorialização, cadastramento da população adscrita e equipe interdisciplinar.

Na atenção básica, o Ministério da Saúde preconiza sejam desenvolvidas ações coletivas e individuais, informando a comunidade, prevenindo a doença, identificando grupos de risco, fazendo o diagnóstico precoce e abordagem terapêutica inclusive a medicamentosa, mantendo o cuidado continuado, educando e preparando portadores e famílias a terem autonomia no autocuidado, prevenindo as complicações, promovendo a saúde para uma melhor qualidade de vida da população adscrita ${ }^{(3)}$.

A retinopatia diabética $(\mathrm{RD})$ é a causa mais frequente de cegueira não infecciosa, e um problema de saúde pública mundial.A prevalência da RD após 15 anos de diabetes varia de $97 \%$ para a DM insulino-dependente e $80 \%$ nos diabéticos não insulino-dependente ${ }^{(4)}$.

O diagnóstico precoce da retinopatia diabética, através de um simples exame de fundo de olho, pode evitar, com medidas terapêuticas sistêmicas e oculares, a progressão da referida doença para formas mais graves, prevenindo, desta forma, a redução da visão ${ }^{(5)}$.

Os objetivos deste estudo foram determinar a prevalência da retinopatia diabética na população cadastrada no Programa de Saúde da Família - Parque Prazeres, em Campos dos Goytacazes e o conhecimento desta doença pelos pacientes diabéticos.

\section{Métodos}

Para a realização deste estudo epidemiológico descritivo transversal, foram selecionados 72 pacientes diabéticos com diagnóstico comprovado através da ficha A de cadastro das famílias do PSF - Parque Prazeres, localizado no município de Campos dos Goytacazes, RJ, em outubro de 2007. Os exames oculares também foram realizados nesta unidade de saúde de atendimento primário que não contava com oftalmoscópio binocular indireto.

Todos os pacientes foram avaliados na unidade com exame de acuidade visual por um único examinador treinado, depois foram submetidos ao procedimento de dilatação pupilar, respondiam a questionário sobre conhecimento das alterações oculares do diabetes e, após a dilatação pupilar era realizado o exame de fundo de olho por oftalmoscopia direta.

A dilatação pupilar foi realizada instilando-se nos olhos do paciente 1 gota dos colírios descritos a seguir na seguinte ordem: inicialmente tetracaína a $1 \%$, após cinco minutos colírio de tropicamida a $1 \%$, repetido após $10 \mathrm{e}$ 20 minutos. Após 30 minutos, colírio de fenilefrina a 10\%.

$\mathrm{O}$ exame de acuidade visual foi realizado conforme protocolo do CBO - Conselho Brasileiro de Oftalmologia nas campanhas de prevenção à cegueira. $\mathrm{O}$ exame de fundo de olho foi realizado de acordo com a metodologia descrita por Yamane ${ }^{(6,7)}$.

Os pacientes assinaram o termo de consentimento, autorizando que o resultado do seu exame fizesse parte de trabalho de pesquisa clínica. O trabalho foi aprovado pelo Comitê de Ética da Universidade Estácio de Sá.

Os dados receberam tratamento estatístico com auxílio do programa Microsoft Excel® e Biostat 5.0®.

\section{Resultados}

Dos 72 pacientes chamados para o exame, compareceram $46(63,9 \%)$. Dos 46 pacientes examinados, 9 ou 19,5\% (IC95\%: 8,1-31\%)) apresentavam retinopatia diabética, dentre estes, 6 ou 13,1\% (IC 95\%:3,3-22,8\%) possuíam RDNP (retinopatia diabética não proliferativa). Uma paciente era portadora de deficiência mental e não cooperou adequadamente com o exame, sendo considerada entre os diabéticos com diagnóstico indeterminado.

Dos 46 pacientes incluídos no estudo, a maioria tinha idade entre 51 a 70 anos (Tabela 1).

Observamos que $78 \%$ dos pacientes examinados eram do sexo feminino; a evolução da doença ocorreu no período de 0 a 10 anos na maioria dos pacientes examinados.

De acordo com questionário aplicado nos pacientes examinados verificou-se que $72 \%$ dos pacientes tinham conhecimento a cerca da possibilidade do diabetes causar problemas visuais, que apenas $20 \%$ sabiam da existência da doença denominada retinopatia diabética e que $89 \%$ dos pacientes relataram não ter qual- 


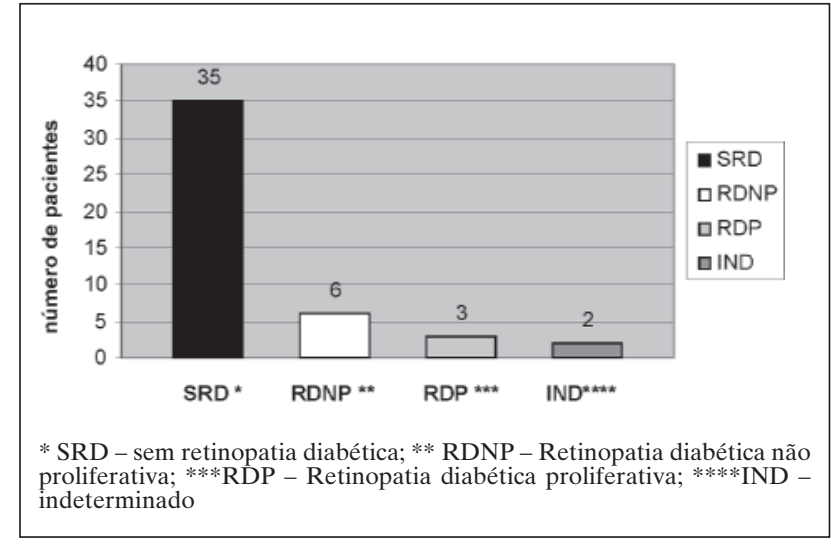

Figura 1: Distribuição da frequência da retinopatia proliferativa e não proliferativa dos pacientes submetidos à oftalmoscopia direta

quer conhecimento sobre exames oculares diagnósticos, porém, $9 \%$ dos pacientes informaram que tinham informações a respeito e dentre estes, $2 \%$ citaram o "laser" e $7 \%$ o fundo de olho. Sessenta e cinco por cento dos pacientes nunca foram submetidos previamente ao exame de fundoscopia.

A frequência anual para exame ocular foi citada como adequada para controle clínico por $26 \%$ dos pacientes. Muitos pacientes (41,3\% ) não sabiam informar a periodicidade necessária de exames para controle clínico da doença (Tabela 2).

Em relação aos cuidados de práticas adotadas pelos pacientes para a prevenção de alterações visuais: $32 \%$ dos pacientes não tinham conhecimento dos cuidados necessários para controle do diabetes, 28,3\% evitavam doces e $10,9 \%$ responderam que era preciso usar medicação adequada (Tabela 3).

Observamos que 38 pacientes $(82,6 \%)$ possuíam visão maior ou igual a 0,3 , destes a maior proporção situava-se entre 0,5 e 1,0 .

Os pacientes com baixa visão ou cegos no momento do exame (visão menor do que 0,3 no melhor olho), que são considerados deficientes visuais no Brasil e pelo critério da OMS (Organização Mundial de Saúde), foram 8 (IC 95\% entre 6,4\% e 28,3\%).

Os pacientes com acuidade visual que apresentaram cegueira, na ocasião do exame, foram 4 ou 8,5\% (visão menor do que 0,05 no melhor olho) pelos critérios da OMS e legislação brasileira.

\section{Discussão}

A Organização Mundial de Saúde (OMS) estimou, em 1997, que após 15 anos da doença: 2\% dos indivíduos acometidos estarão cegos, $10 \%$ terão deficiência
Tabela 1

Distribuição de frequência da idade dos pacientes examinados, no PSF - Parque Prazeres em Campos dos Goytacazes-RJ, 2007

\begin{tabular}{ccc}
\hline Faixa etária & Número de pacientes & $\boldsymbol{\%}$ \\
\hline $20-30$ & 1 & 2,2 \\
$31-40$ & 2 & 4,3 \\
$41-50$ & 7 & 15,2 \\
$51-60$ & 12 & 26,1 \\
$61-70$ & 13 & 28,3 \\
$71-80$ & 9 & 19,6 \\
$81-90$ & 2 & 4,3 \\
TOTAL & 46 & 100 \\
\hline
\end{tabular}

Tabela 2

\begin{abstract}
Distribuição de frequência da periodicidade de fundoscopia considerado adequado pelos pacientes, no PSF - Parque Prazeres em Campos dos Goytacazes-RJ, 2007
\end{abstract}

\begin{tabular}{lcc}
\hline Periodicidade & Número de pacientes & $\boldsymbol{\%}$ \\
\hline Não sabe & 19 & 41,3 \\
Anual & 12 & 26,0 \\
Semestral & 11 & 23,9 \\
Trimestral & 01 & 2,2 \\
Quadrimestral & 01 & 2,2 \\
8 - 8 meses & 01 & 2,2 \\
2 - 2 anos & 01 & 2,2 \\
TOTAL & 46 & 100 \\
\hline
\end{tabular}

Tabela 3

Distribuição de frequência de cuidados pelos pacientes para evitar diminuição visual pelo diabetes, no PSF - Parque Prazeres em Campos dos Goytacazes-RJ, 2007

\begin{tabular}{lcc}
\hline Cuidados & $\begin{array}{c}\text { Número } \\
\text { de pacientes }\end{array}$ & $\%$ \\
\hline Evitar carboidratos & 2 & 4,3 \\
Evitar doces & 13 & 28,3 \\
Glicemia capilar & 3 & 6,5 \\
Medicação & 5 & 10,9 \\
Glicemia capilar / Evitar carboidratos & 1 & 2,2 \\
Evitar carboidratos e doces & 3 & 6,5 \\
Evitar doces e frituras & 1 & 2,2 \\
Evitar doces, frituras e carboidratos & 1 & 2,2 \\
Medicação / Evitar doces & 1 & 2,2 \\
Medicação / Uso de adoçante & 1 & 2,2 \\
Não sabe & 15 & 32,6 \\
TOTAL & 46 & 100 \\
\hline
\end{tabular}




\begin{tabular}{|c|}
\hline ANEXO \\
\hline NODO PRONTUÁRIO: Microárea: \\
\hline IDADE: \\
\hline SEXO: ( ) Feminino ( ) Masculino \\
\hline I. Questionário: \\
\hline 1. Há quanto tempo aproximadamente você tem diabetes? \\
\hline $\begin{array}{llll}\text { ( ) } 5 \text { anos ( ) } 10 \text { anos } & \text { ( ) } 15 \text { anos }\end{array}$ \\
\hline 2. Você sabe que o diabetes pode causar alteração visual? \\
\hline ( ) Sim ( ) Não \\
\hline 3. Você já ouviu falar em retinopatia diabética? \\
\hline ( ) Sim ( ) Não \\
\hline 4. Você conhece algum exame para saber se o olho foi afetado pelo diabetes? \\
\hline ( ) Sim ( ) Não \\
\hline Se a resposta for sim. Qual o exame você indicaria? \\
\hline 5. Você já fez algum exame de fundo de olho? \\
\hline ( ) Sim ( ) Não \\
\hline 6. Você considera este exame necessário? \\
\hline ( ) Não \\
\hline Se disser que sim, de quanto em quanto tempo? \\
\hline 7. Que cuidados poderiam ser tomados para evitar a diminuição visual pelo diabetes? \\
\hline
\end{tabular}

visual grave, 30 a $45 \%$ terão algum grau de retinopatia, 10 a $20 \%$, de nefropatia, 20 a $35 \%$, de neuropatia e 10 a $25 \%$ terão desenvolvido doença cardiovascular ${ }^{(1)}$.

De acordo com Crespo Valdés et al., no estudo realizado sobre a prevalência da retinopatia diabética na atenção primária em Cuba, no período de 1986-2000, houve uma prevalência média de $20,5 \%$ da doença ${ }^{(8)}$.

No estudo realizado por Torres \& Strobl, no Chile, sobre estratégia de detecção da RD em um centro de saúde primária urbano, foram estudados 105 pacientes, e em outro estudo dos mesmos autores, no mesmo ano, em um centro de saúde primário rural, foram estudados 82 pacientes. A prevalência de RD em uma população registrada no centro de saúde primário rural foi de 25,7\% e no centro de saúde urbano foi de $23,5 \%{ }^{(9)}$.

Nosso estudo demonstrou uma prevalência de 19,5\% de retinopatia diabética, que pode ser equiparada aos estudos de Crespo Valdés et al. e de Torres \& Strobl. A semelhança da prevalência indica que o acometimento atingiu $1 / 5$ da população ${ }^{(8,9)}$.
Nosso estudo sobre o conhecimento do diabetes, como causa de alterações visuais, demonstrou que $72 \%$ dos pacientes tinham conhecimento de que a doença causava problemas na visão. Sabe-se que o conhecimento do indivíduo com diabetes mellitus sobre a própria doença é importante, tanto para seu tratamento quanto para a adoção de medidas preventivas, isto pode ser visto no estudo realizado por Pellanda et al. no Brasil. Em outro estudo com dados de 117 pacientes, 56,2\% dos mesmos tinham conhecimento sobre a doença ${ }^{(10)}$. Nosso estudo demonstrou que apenas $20 \%$ dos pacientes sabiam sobre a existência da retinopatia diabética ou tiveram alguma informação.

Segundo Ramos et al., no estudo realizado no departamento de Oftalmologia do Hospital das Clínicas da Universidade Federal do Paraná sobre retinopatia diabética: em uma associação de diabéticos, foram estudados 46 pacientes, onde $80,4 \%$ já haviam tido alguma informação sobre a retinopatia, vê-se que a diferença entre os estudos foi grande. Provavelmente, o grau ins- 
trução de pacientes diabéticos filiados a uma associação de indivíduos com a mesma doença, que se reúnem periodicamente, é maior do que a de indivíduos não filiados em uma comunidade carente ${ }^{(11)}$.

Dentre os 46 pacientes do PSF - Parque Prazeres selecionados, $89 \%$ não tinham nenhum conhecimento sobre exames para avaliação de alterações visuais causadas pelo diabetes. Segundo Silva et al., no seu estudo sobre tratamento da retinopatia diabética: percepções de pacientes em Rio Claro, foram estudados 299 pacientes, destes, 30,8\% desconheciam a doença e 19,7\% consideraram a RD uma doença sem gravidade ${ }^{(4)}$. É necessário ampliar a visão dos pacientes para a prevenção da RD e a formação de grupos de educação e saúde nas comunidades do PSF poderia ajudar a disseminar conhecimentos sobre a doença.

Dos 46 pacientes estudados no PSF - Parque Prazeres, $65 \%$ não foram submetidos previamente ao exame de fundoscopia. No estudo realizado por Ramos et al., 65,2\% nunca fizeram fundoscopia e cerca de 46,1\% já haviam sido submetidos pelo menos a um exame ${ }^{(11)}$. Muitos não sabem que são portadores da RD e quando é realizado o diagnóstico a doença se encontra no estágio avançado ${ }^{(9)}$. Por isso, é importante os exames periódicos de fundoscopia e para viabilizar este exame, faz-se necessário implantá-lo como rotina no PSF.

Os cuidados para evitar a diminuição visual pelo diabetes não foram conhecidos por $32,6 \%$ da amostra de 46 pacientes estudados no PSF - Parque Prazeres. Segundo Sherwin, a terapia clínica limita-se ao controle da glicemia, que retarda a evolução da RDNP, bem como o controle da hipertensão que são preventivos e reduzem a progressão da retinopatia diabética ${ }^{(12)}$.

A medida da acuidade visual do melhor olho, de 18 pacientes neste estudo foi maior ou igual a 0,8 . No estudo de Mendez Sanchez et al., em cerca de 140 (68,9\%) olhos examinados, a acuidade visual variou entre 1,0 - 0,8, num total de 203 pacientes estudados ${ }^{(13)}$.

Dentre os 46 pacientes (92 olhos) selecionados no PSF - Parque Prazeres, submetidos ao exame de fundoscopia, 6 (13,0\%) pacientes apresentaram RDNP, $3(6,5 \%)$ dos pacientes apresentavam RDP e sem RD foram 35 (76\%). Os autores Torres e Strolb após realizar um estudo sobre prevalência da retinopatia diabética em uma população registrada em um centro de saúde primário rural, no Chile, observaram em 82 pacientes que 61 pacientes não tinham RD (74,4\%), 18 pacientes $(22 \%)$ tinham RDNP e 3 pacientes $(3,7 \%)$ tinham $\mathrm{RDP}^{(9)}$. A diferença entre os dados percentuais dos dois estudos variou somente em $2,4 \%$, entre os pacientes sem RD.
Durante esta pesquisa, não encontramos após extensa busca na literatura, trabalhos científicos sobre detecção da RD no PSF. Portanto, seria útil novos trabalhos para avaliar o conhecimento dos profissionais de saúde do PSF sobre RD.

A retinopatia diabética é um problema de saúde pública em muitos países do mundo e muitos profissionais de saúde receberam informações sobre a RD na atenção básica, mas não possuem qualquer experiência prática em relação aos cuidados do PSF. Em outros países, há estudos variados na atenção primária, tendo a retinopatia diabética como uma das ações preventivas prioritárias $^{(8-10,13)}$.

\section{Conclusão}

Programas de prevenção a cegueira pela retinopatia diabética no PSF estão justificados se a prevalência desta doença nas comunidades carentes assistidas manter-se em níveis elevados (19.5\%), como os observados neste trabalho.

A melhora do conhecimento dos pacientes sobre o risco de perda de visão e exames oftalmológicos poderá contribuir para melhor controle da retinopatia diabética.

\section{Abstract}

Objective: Determine the diabetic retinopathy prevalence in a Family's Health Program unity in Parque Prazeres - Campos dos Goytacazes, RJ, and the level of patient's knowledge about diabetes visual impairment. Methods: Seventy two diabetic patients were selected, but only 46 attended the research. The patients were chosen based on Family's Health Program medical registers. They were submitted to visual acuity examination and afterward to a questionnaire in order to evaluate their knowledge level about visual impairment. Then, they were submitted to a direct fundoscopic examination. Statistical studies were done based on Microsoft Excel and Bioestat 5.0 Results: Diabetic retinopathy prevalence was about 19,5\% (CI $95 \%$ between 8,1 and $31 \%$ ); $13 \%$ showed non proliferative diabetic retinopathy and 6,5\% showed proliferative retinopathy. In accordance with World Health's Organization criteria: 8,5\% were considered blind and 8,9\% with low vision at the moment of ocular examination. Seventy two percent of patients had previous knowledge that diabetes could cause visual reduction, but $80 \%$ didn't know the necessary exams to diagnose it and $65 \%$ never were previously submitted to ophthalmoscopy. Conclusion: Blindness prevention programs by diabetic 
retinopathy in Family Health Programs are justified considering that the retinopathy diabetic prevalence in assisted communities could be high, as was in this study. The improvement of patient knowledge about the vision threatening risk and ophthalmological exams can allow a better retinopathy diabetic control

Keywords: Diabetic Mellitus/complications; Diabetic Retinopathy; Family health program; Blindness/ prevention \& control; Diagnostic techniques, ophthalmological; Ophthalmoscopy/methods.

\section{REFERÊNCIAS}

1. Brasil, Ministério da Saúde. Secretaria de Atenção a Saúde. Departamento de Atenção Básica. Diabetes mellitus. Brasilia: Ministério da Saúde; 2006. [Caderno de atenção básica; 16. Série A. Normas e manuais técnicos].

2. Organização Panamericana de Saúde. Doenças crônicodegenerativas e obesidade: estratégia mundial sobre alimentação saudável, atividade física e saúde. Brasília: OPAS/OMS; 2003.

3. Norma Operacional da Assistência à Saúde. NOAS-SUS 01/ 2001.Informe da Atenção Básica [Internet]. 2001:2(12).[citado 2008 Nov 15].

Disponível em:http://dtr2004.saude.gov.br/dab/docs/ publicacoes/informes/psfinfo12.pdf

4. Silva VB, Temporini ER, Moreira Filho DC, Kara-José N Tratamento da retinopatia diabética: percepções de pacientes em Rio Claro (SP) - Brasil. Arq Bras Oftalmol. 2005; 68 (3): $363-8$
5. Powers A. Medicina interna. In: Braunwald E; Fauci AS, Kasper DL, Hauser SL, Longo DL, Jameson JL, editores. Medicina Interna. $15^{\mathrm{a}}$ ed. Rio de Janeiro: Mc Graw-Hill; 2002.

6. Conselho Brasileiro de Oftalmologia [Internet]. Departamento de Oftalmologia da Associação Médica Brasileira. DoençasRetinopatia diabética. [citado 2006 Jun22]. Disponível em: http:/ / w w w c c bo.com.br/pacie n tes/doencas/ doencas_retinopatia_diabetica.htm

7. Yamane R, editor. Semiologia ocular. $2^{\mathrm{a}}$ ed. Rio de Janeiro: Cultura Medica; 2003. p. 367.

8. Crespo Valdés N., Padilla González JC, González Fernándes R, Crespo Mojena N, Rodríguez Casales M, Hernández Beguiristain JD. et al. Prevalencia de la retinopatía diabética en pacientes del nivel primario de salud. Rev. Cuba Med Gen Integr. 2004; 20 (2): 40-5.

9. Torres AC, Strobl VA. Estrategia de detección de retinopatía diabética en un centro de salud primario. Arch. Chil. Oftalmol. 2003; 60 (1): 47-52.

10. Pellanda LC, Canani LHS, Zimmer PM, Karohl C, Hidalgo PM, Silveira SM, Schmidt MI. Grau de conhecimento sobre diabetes mellitus: um estudo populacional. Rev AMRIGS. 1989; 33(4): 322-6.

11. Ramos S R, Sabbag F P, Busato D, Miranda A B, Moreira Junior C A. Retinopatia diabética: estudo de uma associação de diabéticos. Arq Bras Oftalmol.1999; 62(6):735-7

12. Sherwin, RS. Diabetes Melito. In: Goldman L, Bennet JC, editores. Cecil: Tratado de medicina interna. $21^{\mathrm{a}}$ ed. Rio de janeiro: Guanabara Koogan; 2001 p 1405-1430

13. Mendez Sanchez TJ, Lopez Cardet R, Rosales Quinones C, Seuc Jo A. Prevalencia de retinopatia diabetica en un area de salud del Município de Marianao. Rev Cuba Oftalmol.1996; 9 (2): 116-21. 